\title{
Modelling of Dynamic Voltage Restorer against Balanced and Unbalanced Voltage Sags in Distribution systems
}

\author{
H. Lakshmi \\ Electrical and Electronics Engineering, Kakatiya University, Warangal, India.
}

\begin{abstract}
The Dynamic Voltage Restorer (DVR) is used to regulate the voltage at the load terminals from various power quality problems like sag, swell, harmonics, unbalance etc. in supply voltage. This paper presents modelling aspects of several types of Dynamic Voltage Restorer (DVR) working against various voltage sags by simulation. Dynamic voltage restorers (DVRs) are used to protect sensitive loads from the effects of voltage sags on the distribution feeder. Significant simulation results show that these several types of the modelled device can work very well against balanced and/or unbalanced voltages caused by faults in a distribution system. Detailed analyses illustrate that with suitable parameter setting these devices can deal with different levels of voltage sag severity. In addition, appropriate ways to obtain a good quality output voltage by a DVR during voltage sag is also presented. It then provides analyses of working performance of the device, including capability and quality of compensation.
\end{abstract}

Index Terms- Dynamic Voltage Restorer (DVR), Balanced and unbalanced faults, Compensation capability, Power quality sag severity, voltage sag.

\section{Introduction}

Power quality (PQ) is a term which has captured increasing attention in power engineering within the recent years. For most of the electric power engineers, the term refers to a certain sufficiently high grade of electric service. Usually the term quality refers to maintaining a sinusoidal waveform of bus voltages at rated voltage and frequency [1].

One of the fundamental challenges facing utility and working staff is the need to become familiar with and stay informed about issues dealing with power quality. Power suppliers and also the customers are going to find a solid background in power quality not only useful, but necessary too, for continued productivity and competitiveness. These facts are supported by the utility industry which undergoes restructuring and as customers find their service needs changing with increased use of equipment and processes more susceptible to power system disturbances.

Power quality is a growing concern for a wide range of customers. Industrial customers can experience interruptions of important processes during momentary voltage sags associated with faults within the utility system [2]. Commercial customers are installing high efficiency lighting and electronic office equipment, resulting in higher harmonic levels in the buildings. These harmonic sources cause excessive neutral currents and transformer overheating. Even residential customers are concerned about surge protection for sensitive electronics in the home and the impact of momentary interruptions on their electronic equipment.

Power quality within the electric distribution system is a growing concern. Customers require higher quality service due to more sensitive electronic and computer-controlled loads. Capacitor switching events and voltage sags associated with remote faults that never caused problems in the past, now cause equipment tripping and even failures within customer facilities. Also, customer loads are generating increasing amounts of harmonic currents that can be magnified on the distribution system due to resonance conditions [3].

As technology becomes more advanced, equipment has become more sensitive to fluctuations in voltage along the distribution line. New equipment that increases productivity for a plant may also cause power quality problems for other equipment down the line [4]. Power quality is now viewed from a systems perspective rather than as an isolated instrument problem. Understanding the entire scope of the problem helps in identifying the solution and preventing future occurrences.

The existence of disturbances requires analysis, monitoring and taking measures to ensure the quality of electricity. Therefore, disturbances are those that significantly reduce the quality of electricity affecting the generation, trans-mission and distribution process, but also the electricity consumption [2].

Voltage is the main qualitative element that conditions the proper functioning of the receptor. That is why the voltage quality practically defines the power quality. The paper is focusing on the power quality monitoring in case of an important substation within the Romanian Power System.

One of the major concerns in electricity industry today is power quality problems to sensitive loads. This is due to the advent of a large numbers of sophisticated electrical and electronic equipment, such as computers, programmable logic controllers, variable speed drives, and so forth. The use of these equipment's 
very often requires power supplies with very high quality. Voltage sag, which is a momentary decrease in rms voltage magnitude in the range of 0.1 to 0.9 per unit (p.u.) [5], is considered as the most serious problem of power quality. It is often caused by faults in power systems or by starting of large induction motors. It can interrupt or lead to malfunction of any electric equipment which is sensitive to voltage variations. It occurs more frequently than any other power quality phenomenon does. Therefore, the loss resulted due to voltage sag problem for a customer at the load-end is huge.

Dynamic voltage restorer (DVR) and static compensator (STATCOM) are recently being used as the active solution for voltage sag mitigation

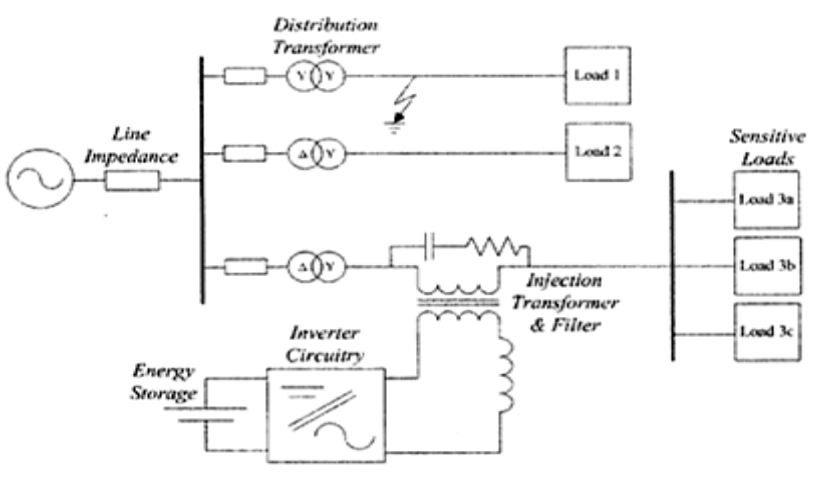

Fig. 1. Typical location of a DVR in a power network.

These system-equipment interface devices are commonly known as custom power devices [6], in which DVR is a powerful one for short-duration voltage compensation. Unlike the STATCOM which connects to the load in parallel, the DVR is connected in series with the load hence it possesses some certain advantages.

For example, during power disturbances DVR installed in front of a critical load will appropriately provide correction to that load only. It is noteworthy that during normal operation due to the series connection a DVR may have to provide a small amount of voltage drop mainly at the coupling transformer. Also DVR cannot provide compensation during full power interruptions.

\section{Voltage Sags}

Voltage sags are one of many power quality related problems the industrial process sector has to face [7], [8], though sags are one of the most severe.

Voltage sags are defined as short duration reductions in the rms supply voltage that can last from a few milliseconds to a few cycles, with typical dip depths ranging from 0.9 to $0.5 \mathrm{pu}$ of a 1 -pu nominal. It has been shown that year on year voltage sags cause extensive disruption to the industrial process sector in terms of production loss [7], [9], which make them a particularly important area.

There are various solutions to this problem, examples being: Designing inverter drives for process equipment to be more tolerant of voltage fluctuations or the installation of voltage correction devices. It has already been shown [7] that for customers of large loads, from the high kilowatt to the low megawatt range, a good solution is the installation of a dynamic voltage restorer (DVR); see Fig. 1.

A DVR is primarily for use at the distribution level, where the basic principle is to inject a voltage in series with the supply when an upstream fault is detected. Loads connected downstream of the DVR are thus protected from any voltage sags caused by faults elsewhere on the network.

The location of the DVR, in terms of the connection arrange-ment of upstream transformers (typically) and the type of protection it is to offer to potentially sensitive loads, is a major factor when determining the type of inverter control required. The main DVR control used in conjunction with the sag detection techniques presented in this paper utilizes a type a vector control that only considers the positive and negative sequence information in the supply. The DVR is located downstream of a delta-star distribution transformer (Fig. 1), thus eliminating the need to control the zero sequence.

\section{A. Modelling in PSCAD}

\section{Dvr Against Balanced Volatge Sags}

This section will briefly highlight one way of modelling a DVR in PSCAD against balanced voltage sags based on published literature and show the result of mitigation obtained.

There are typically four main components to model a DVR

[3]:

- Coupling transformer 
- DC voltage source

- Multi-pulse bridge inverter

- Control system

A typical DVR built in PSCAD and installed into a simple power system to protect a sensitive load in a large radial distribution system [10] is presented in Fig. 2. Its control system block diagram is shown in Fig. 3. The coupling transformer with either a delta or wye connection on the DVR side is installed on the line in front of the protected load. Filters can be installed at the coupling transformer to block high frequency harmonics caused by DC- to-AC conversion to reduce distortion in the output [11]. The DC voltage source is an external source supplying DC voltage to the inverter to convert to AC voltage. The optimisation of the DC source can be determined during simulation with various scenarios of control schemes, DVR configurations, performance requirements, and voltage sags experienced at the point DVR is installed.

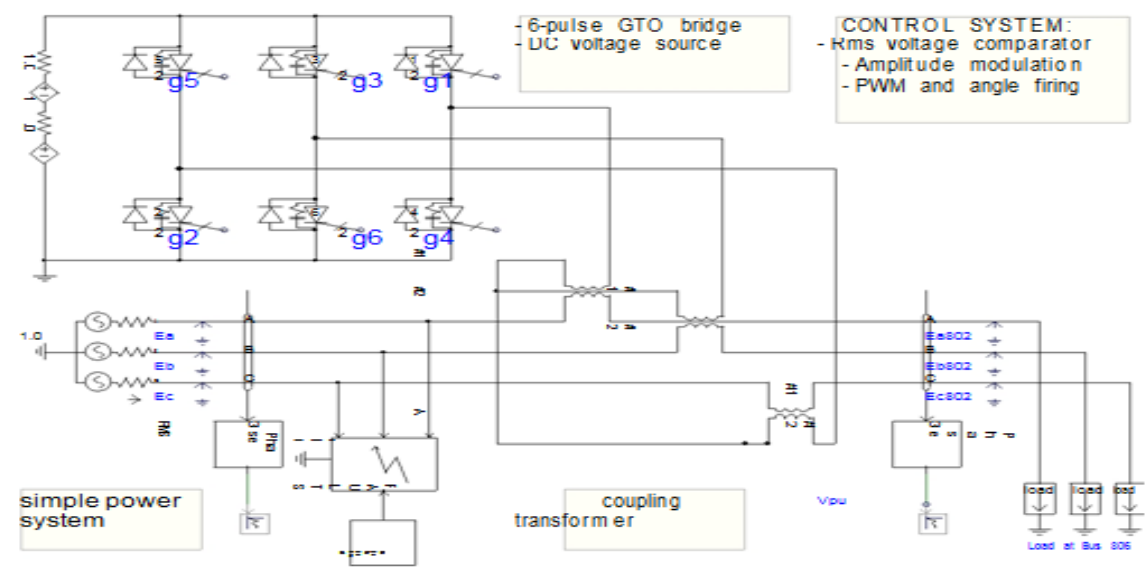

Fig. 2 DVR with main components in PSCAD

The inverter is a six-pulse gate turn off (GTO) thyristor controlled bridge. Currents will follow in different directions at outputs depending on the control scheme, eventually supplying AC output power to the critical load during power disturbances. The control of this bridge is indeed the control of thyristor firing angles. Time to open and close gates will be determined by the control system. There are several methods for controlling the inverter. To model a DVR protecting a sensitive load against only balanced voltage sags, a simple method of using the measurement of three-phase rms output voltage for controlling signals can be applied. Amplitude modulation (AM) is then used. In addition, to provide appropriate firing angles to thyristor gates the switching control using pulse width modulation (PWM) technique and interpolationfiring [6] is employed.

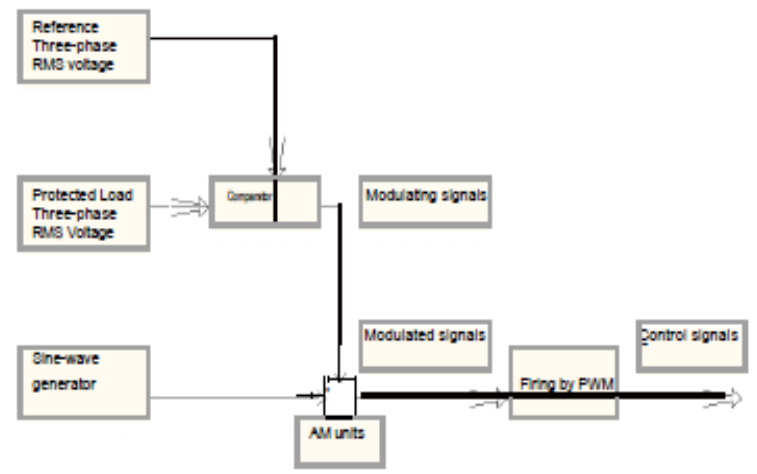

Fig. 3 DVR Control system block diagram

The following two figures illustrate two different levels of voltage sags that the DVR works with. Each figure shows the rms voltages before and after DVR is connected during voltage sag. As can be clearly seen, irrespective of the voltage sag levels the output voltages recover back closely to reference value, which means that the DVR can cope well with voltage sag in both cases. 


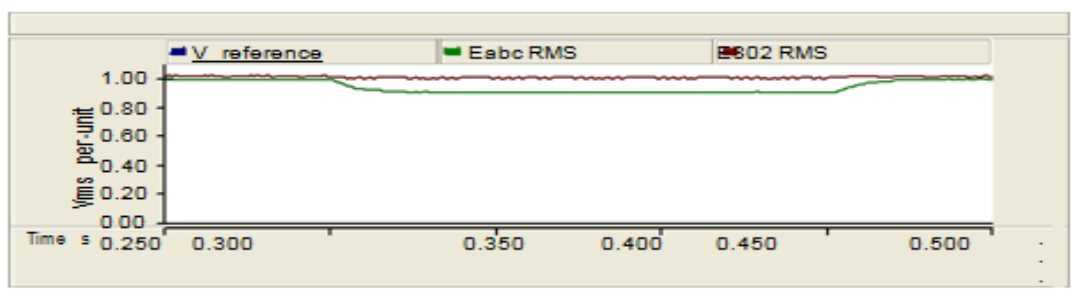

Fig. 4 The source (Eabc RMS) and protected load (E802 RMS) rms voltages in a shadow sag

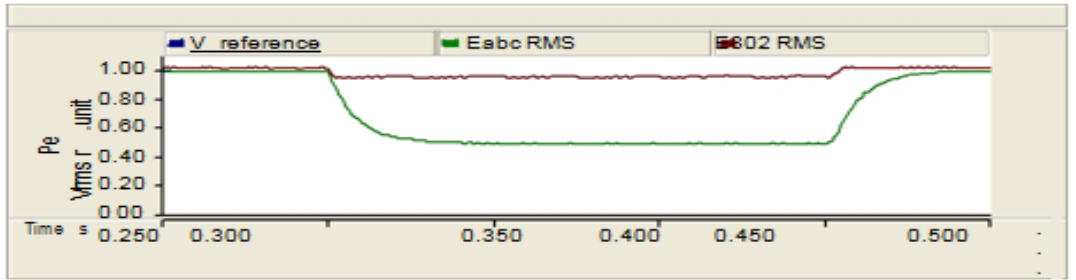

Fig. 5 The source (Eabc RMS) and protected load (E802 RMS) rms voltages in severe sag

When the actual load rms voltage is set higher than the reference value, DVR also works to recover it back. In other words DVR should cope with voltage swell as well.

Various simulations showed an excellent performance of this DVR to work against balanced voltage sags and swells. The outstanding problem is that this type of DVR can only deal with balanced voltage sags.

\section{A. General}

\section{Dvr Against Balanced And Unbalanced Voltage Sags}

Unbalanced voltage sags are very common in distribution systems. Voltages sags are normally caused by remote faults, either within the distribution systems or downward from transmission lines. While the three phase balanced fault is often seen as the worst case, it seldom occurs. The most frequent types of faults include Single phase to ground fault (SLGF) and Line to line fault (LLF), which can be grounded or non-grounded. In distribution systems $95 \%$ of faults are unbalanced type. They result in unbalanced sags.

In the previous section the DVR detects 3-phase rms voltage of the critical load and then employs PWM technique to control the firing of GTO's to pump up DC voltage to all three phases of load

\section{B. Mitigation Result}

An almost worst-case fault (local, three-phase-to-ground, and with a small impedance) by lightning strikes was made as shown in Fig.2. The line impedance has been taken out for simulation comparison purposes, which does not have any effect to the observed result. The load is a fixed load and not a motor type.

To satisfy these three criteria rms voltages can still be measured for differential control signals but they have to be obtained from single-phase rms measurement not from three-phase. The control signals will be separately made for three phases A, B, and C. Besides, modification of DVR inverter bridge and transformer connection should be made and then return without affecting the other two phases. It is noted that to maintain an equal injecting voltage to each phase, the same value of DC voltage at each half of the source would be required. In Fig. 7 instead of having only one three-phase rms voltage three separate line-to-neural (L-N) values are now used for firing control input. Three groups of GTO's are now working independently from each other's. They start firing in case their corresponding phase rms voltage is different from one. When all the phase voltages of the load reach to desired value the control system stays at stable condition. Other properties of the DVR shown in section I are kept unchanged. Consequently, the three criteria for DVR working in both unbalanced and balanced voltage sags are obtained. Result of mitigation against various voltage sags are shown in Figs. 8,9 and 10 accordingly. Most importantly, the changes should not affect the performance of the DVR against balanced sags described previously because the objective of building DVR is that it should be able to deal with all kinds of voltage sags.

Two methods of coupling transformer configuration were investigated: a wye-connected with grounding point and a delta-connected between phases. To adapt to each of these methods the control systems were appropriately amended. In fact, two different single-phase rms voltage detection schemes were established for each method. One is a detection of voltages between line to neutral for the first and the other is that between line to line for the second respectively. The following part will describe configuration of the wye-connected 
method and its results of voltage sag compensation. Three different types of faults with the same fault impedance for comparison purposes will be created in the order: SLGF, LLF (grounded) and three-phase-toground fault.

\section{B. The Wye-connected DVR}

The connection diagram of DVR in PSCAD is shown in Fig.6. Its block diagram of the modulating signals in control system is shown in Fig.7.

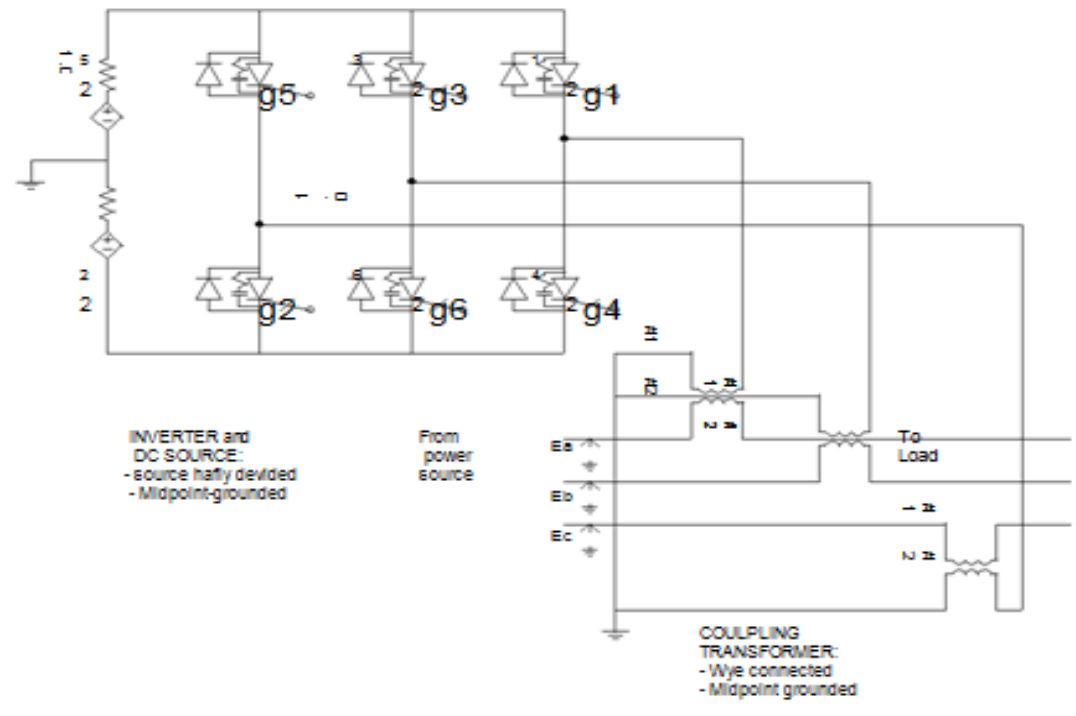

Fig.6 DVR configuration diagram - Wye-connected method

In Fig. 6 the transformer is wye-connected with a common connection to the midpoint of the DC source. This allowsthatcurrentwillpumpintoeachphasethrougheachpairofGTOvoltage three separate line-to-neural (L-N) values are now used for firing control input. Three groups of GTO's are now working independently from each other's. They start firing in case their corresponding phase rms voltage is different from one. When all the phase voltages of the load reach to desired value the control system stays at stable condition. Other properties of the DVR shown in section I are kept unchanged. Consequently, the three criteria for DVR working in both unbalanced and balanced voltage sags are obtained. Result of mitigation against various voltage sags are shown in Figs. 8,9 and 10.

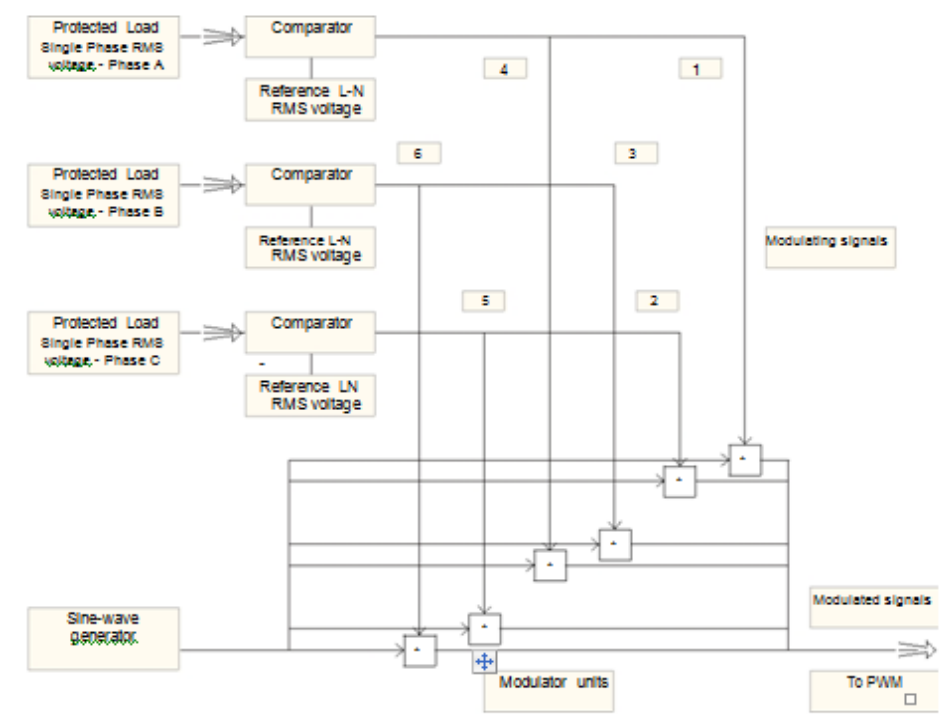

Fig. 7 Control block diagram - Wye-connected method 


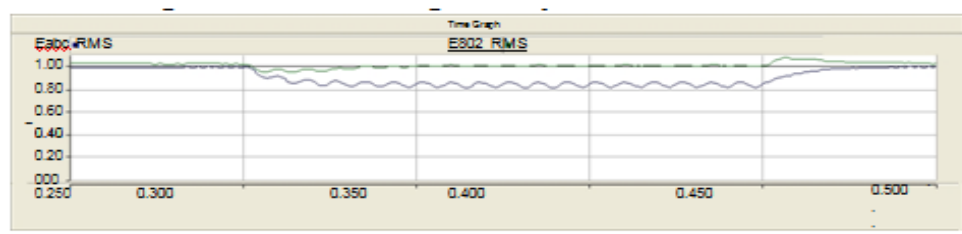

Fig. 8 The source and protected load rms voltages of faulted phase in a SLGF

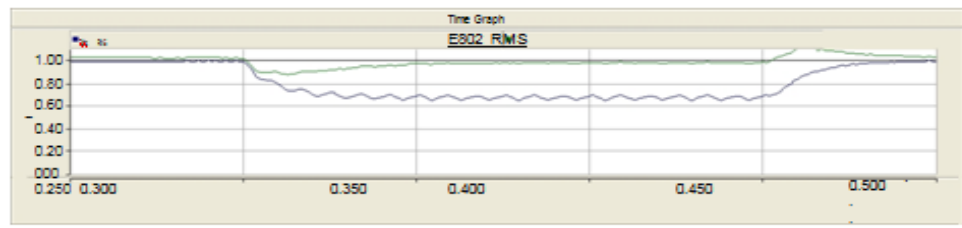

Fig. 9 The source and protected load rms voltages of faulted phase in a LLGF

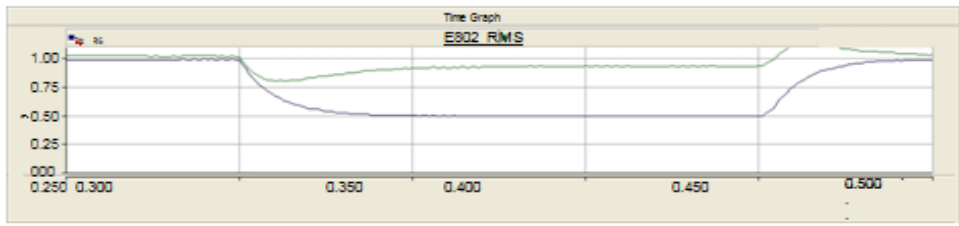

Fig. 10 The source and protected load rms voltages in a three phase fault

In Fig. 8, a fault causes rms voltage (phase A) down to about $80 \%$. As can be seen, the rms voltage of the protected

load stays the same at around unity. The other healthy phase voltages which are not shown here still remain constant. Fig. 8 shows that the line-to-line fault results in a more severe voltage sag (down to about $65 \%$, phase A), compared to that of single phase. Regardless of the sag magnitude critical load voltage notices a good compensation. Meanwhile with the worst three-phase voltage sag down to half of the nominal value critical load voltage the mitigation equipment still provides a good compensation. This newly simulated DVR shows a similar performance for voltage sag compensation with that of the DVR which uses the threephase rms measurement method. The response time of this DVR with three phase voltage measurement is a bit slower than the previous one. Still this detection and compensation time to increase rms voltage up to desired value is within couple of cycles, proving that DVR can work fast enough that would not result in equipment trouble if any severe sag occurs.

As can be seen single-phase monitoring method gives a less stable rms voltage curve compared to the three-phase case making it difficult to keep an appropriate signal for control. That involves the rms smoothing time constant, which is a constant interval time applied for detection of a considerable differential signal to control the compensation process. This value on the one hand must be small enough for the system to be able to detect voltage sags. On the other hand the penalty is a huge fluctuation in rms value record that can lead to wrong differential signal for firing control.

By applying extensive simulations when varying this detection time in PSCAD it can be concluded that the method can be satisfactorily employed for DVR using in systems with three phase balanced sags requiring measuring the three-phase rms voltage. Besides, the method can also be applied for systems with unbalanced sags using single-phase rms voltage measurement. In the second case appropriate modifications may need to be done to keep the latter to cope with worst case scenarios of voltage sag.

\section{Analysis Of Dvr Compensation Capability \& Quality Performance}

In a DVR, there are two main considerations in its working performance: the compensation capability and the output voltage quality. In a STATCOM, there is some influence of the capacitor size on its performance, including distortion. It is revealed by simulation that the value of the DC source voltage has also some side-effect as distortion to the output waveform. In addition, the severity of sag decides whether or not the DVR with that set value of DC voltage is capable of compensating. In other words, there are two main factors relating to the capability and performance of DVR working against voltage sags in a certain power system: the sag severity level and the total harmonic distortion (THD) introduced to protected load. Both of these in turn are mainly decided by the DC source. The study of relation among these three factors enables to select appropriate parameters for DVR to cope with worst-case scenarios of voltage sag. Thus, in this section, an analytical method of examining the changes of harmonic distortion and compensation capability according to the changes of DC source voltage is 
presented. Based on that, considerations on DVR parameter setting and voltage sag compensation strategies can be addressed.

\section{A. Compensation Capability}

By definition [1], a value of rms voltage recovery greater than $90 \%$ nominal value is acceptable. The issue of how much improvement in rms voltage after compensation is also dependent on the DC voltage value. For example, the sag in the above case is corresponding to a new curve of load voltage (E802rms) as shown in Fig. 11

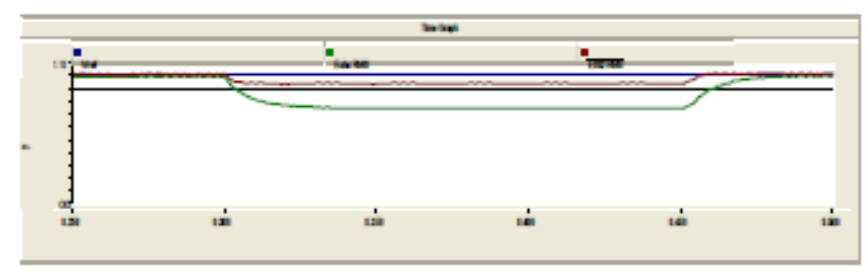

Fig. 11 Improvement in voltage sag compensation

Apparently, the load voltage value goes up to about 95\%, which satisfies the above criterion. This curve would change if the DC source value is changed too.

\section{B. Simulation Results}

In this work, the DVR against balanced voltage sags described in section II is applied. For the two types described in section III, the same procedures can be done. Various three-

phase faults with different sag severity are created. First, a constant value of DC source voltage is set and the changes in total harmonic distortion and load rms voltage due to voltage sag severity changes is recorded. Then, it is repeated for a number of other DC voltage values. The results simulated are summarized in tables I and II.

The two tables correspond to data recorded in two different severe sags. In each table, the first column shows variation of DC source. The second and the third columns record the values of voltage after compensation and THD according to this DC source variation. It is noted that for comparison purposes, the definition of voltage sag by how many percent is now applied. Moreover, THD in percentage is a constant for each case. This is because the average value of THD is applied. In fact, in Fig. 10, THD is actually not a constant value before, during and after fault. However, the increase in THD at the points where the fault occurs and cleared is quite short. Meanwhile, harmonic is more significant when it appears in a fairly longer time, as it is the factor which mainly causes non useful heat dissipation in load equipment and so forth. The last two columns in each table present the derived thresholds that voltage sag and THD must meet. Similar tables of results can be obtained for other voltage sags having different severity. The results in tables I and II are converted into two graphs shown in Fig.12 and Fig.13.

Table I A severe voltage sag - voltage drops by $50 \%$

\begin{tabular}{|c|c|c|c|c|}
\hline $\begin{array}{c}\text { DC } \\
\text { voltage }\end{array}$ & $\begin{array}{c}\text { Load } \\
\text { voltage }\end{array}$ & $\begin{array}{c}\text { THD } \\
\text { threshold }\end{array}$ & $\begin{array}{c}\text { Sag } \\
\text { threshold }\end{array}$ & THD \\
\hline$(\mathrm{kV}):$ & $(\%)$ & $(\%)$ & $(\%)$ & $(\%)$ \\
\hline 0 & 50 & 0 & 10 & 5 \\
\hline 5 & 35 & 4 & 10 & 5 \\
\hline 10 & 20 & 2.5 & 10 & 5 \\
\hline 15 & 10 & 2 & 10 & 5 \\
\hline 20 & 6 & 2 & 10 & 5 \\
\hline 25 & 4 & 3 & 10 & 5 \\
\hline 30 & 2 & 4 & 10 & 5 \\
\hline 35 & 0 & 5 & 10 & 5 \\
\hline
\end{tabular}


Table II. Very severe voltage sag - voltage drops by $75 \%$

\begin{tabular}{|c|c|c|c|c|}
\hline $\begin{array}{c}\text { DC } \\
\text { voltage }\end{array}$ & $\begin{array}{c}\text { Load } \\
\text { voltage }\end{array}$ & $\begin{array}{c}\text { THD } \\
\text { threshold }\end{array}$ & $\begin{array}{c}\text { Sag } \\
\text { threshold }\end{array}$ & THD \\
\hline$(\mathrm{kV}):$ & $(\%)$ & $(\%)$ & $(\%)$ & $(\%)$ \\
\hline 0 & 75 & 0 & 10 & 5 \\
\hline 5 & 60 & 8 & 10 & 5 \\
\hline 10 & 45 & 10 & 10 & 5 \\
\hline 15 & 30 & 9 & 10 & 5 \\
\hline 20 & 15 & 3.5 & 10 & 5 \\
\hline 25 & 9 & 3 & 10 & 5 \\
\hline 30 & 6 & 4 & 10 & 5 \\
\hline 35 & 5 & 5 & 10 & 5 \\
\hline
\end{tabular}

\section{Result Analysis}

The results shown in Tables I and II are converted into two corresponding graphs shown in Fig. 12 and Fig. 13.

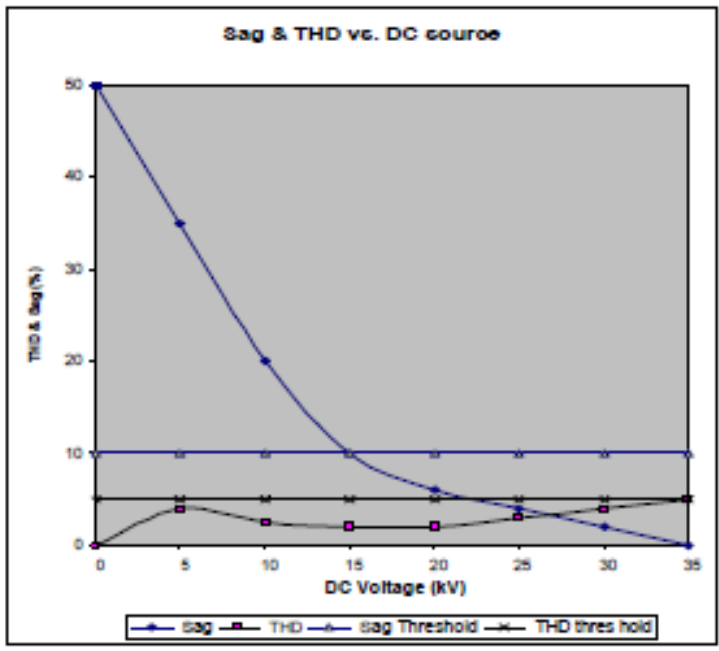

Fig. 12 The severe voltage sag - voltage drops by $50 \%$

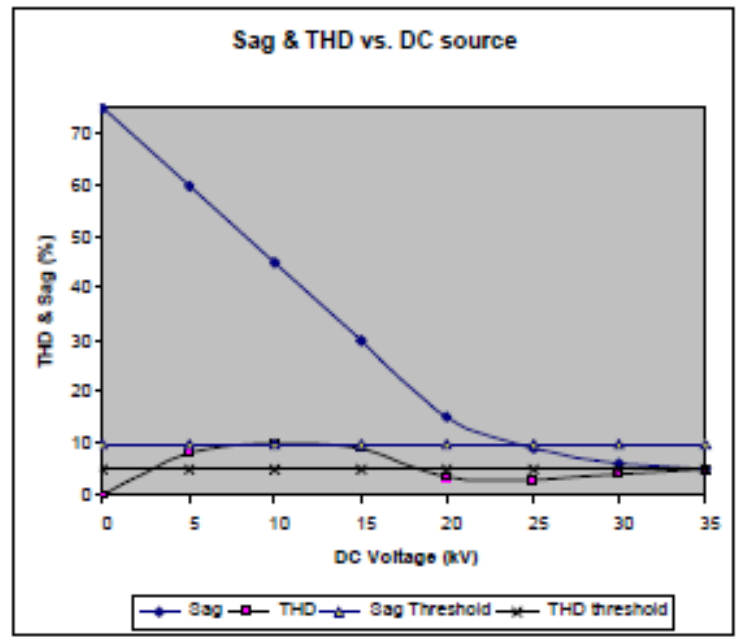

Fig. 13 The very severe voltage sag - voltage drops by $75 \%$

As can be seen, the graphs give a very clear explanation for which range of DC value source that would satisfy the DVR operation requirement in each case. Depending on the worst-case level of the sag in the system that DVR is required to be capable to compensate, the DC voltage value can be appropriately selected. 
For example, if it is required that DVR should be robust enough to cope with balanced sag with value down to $50 \%$ at the point of installation; it is corresponding to the case in the first graph. In this one, the sag threshold $(10 \%)$ cuts the load voltage sag curve at a point corresponding to a DC voltage value of $15 \mathrm{kV}$. It is noticed that the curve is relatively linear. These mean that with any value DC voltage value greater $15 \mathrm{kV}$, the DVR is able to cope with such severe voltage sag (50\%). Similarly for the harmonic curve, a less than $35 \mathrm{kV}$ DC voltage would result in a compensation in which harmonic distortion is within limit (5\%). Thus, to cope with the above sag compensation and harmonic distortion requirements, a DC source voltage raging from $15 \mathrm{kV}$ to $35 \mathrm{kV}$ should be selected for this DVR.

It is important to note that the shape of THD curves in the above figures will change if a higher number $\mathrm{N}$ is selected. This explains why increasing DC source value does not result in a dramatic increase of THD, as seen in the graphs. However, higher frequency harmonics can be easily removed by filters as mentioned, they are not of important. In the case that the full impact of the harmonic distortion introduced to the load needs to be considered, the range of DC source value found would be changed accordingly. Still the selection principle remains unchanged.

It can also be seen that while the sag curves are nearly linear, those of harmonics are not. This implies that increasing DC source voltage may improve the capability that DVR can cope with more severe sags; however, depending on the firing control scheme and other factors, harmonics injected into power system may differently vary.

With a requirement of different voltage sag level, another range of the source value can be obtained. Generally speaking, the best case that a DVR may need is it can cope with a local and bolted balanced fault. By proceeding in the same way, a suitable value of DVR might be selected. In that case, DVR is said to be able to work with all levels of balanced voltage sag severity.

When DC voltage is large enough but harmonic distortion requirement does not meet, there are other ways to change DVR parameters that can utilise a better harmonic performance. These include changing in PWM technique and installing filter at coupling transformer as mentioned, and so forth. Consequently, the criteria that decide the DVR capability and output quality stated earlier can be obtained.

\section{Conclusion}

Several aspects of voltage sag mitigation study have been examined. First, a DVR using six-pulse inverter and three-phase rms voltage measurement and sine wave PWM control was described. It presents excellent performance to protect critical loads against balanced voltage sags. Then, a DVR using singlephase rms voltage measurement that works very well against not only balanced voltage sags but also unbalanced ones resulting from both single-line and line-line faults was presented. Finally, the study of DVR capability and quality performance was examined thoroughly. This discusses appropriate ways to configure DVR so that it can deal with all types of voltage sag - balanced and unbalanced, and with all levels of sag severity shadow, severe and worst. This addresses the harmonic distortion problem that DVR produces in the power system. The whole study was mainly involved with changes in the value of DC source voltage in DVR.

A full simulation of DVR mitigation in a large radial system (IEEE 34-bus distribution feeder [4]) was performed in PSCAD. This has not been presented here due to space constraints but will be presented in a future paper.

\section{References}

[1] T.L. Tan, S. Chen, and S.S. Choi, "An overview of power quality state estimation", the $7^{\text {th }}$ International IEEE Power Engineering Conference, (IPEC05), 2005, pp: 1-276.

[2] G Putms, J. Wijayakulasooriya, and P. Minns, "Power Quality: Overview and monitoring", IEEE International Conference on / Industrial and Information Systems (ICIIS07), 2007, pp: 551-558.

[3] T Vmnal, K. Janson, and H. Kalda, "Analysis of power consumption and losses in relation to supply voltage quality", IEEE $13^{\text {th }}$ European Conference on Power Electronics and Applications EPE'09,2009, pp: 1-9.

[4] R. Lima, D. Quiroga, C. Reineri, and F. Magnago, "Hardware and software architecture for power quality analysis." Computers \& Electrical Engineering, Vol. 34 (6), pp. 520-530,2008..=

[5] IEEE recommended practice for evaluating electric power system compatibility with electronic process equipment, IEEE Standard 1346-1998. 1998.

[6] N.H. Woodley, L. Morgan, and A. Sundaram, "Experience with an inverter-based dynamic voltage restorer", IEEE Trans. Power Delivery, vol. 14, issue 3, pp. 1181 -1186, Jul. 1999.

[7] M. H. J. Bollen, Understanding Power Quality Problems: Voltage Sags and Interruptions. New York: IEEE Press, 1999.

[8] M. F. Mc.Granaghan, D. R. Mueller, and M. J. Samotyj, "Voltage sags Q ${ }^{\text {in }}$ industrial systems," IEEE Trans. Ind. Applicat., vol. 29, pp. 397403, Man/Apr. 1993.

[9] J. C. Smith, J. Lamoree, P. Vinett, T. Duffy, and M. Klein, "The impact of i voltage sags on industrial plant loads," in Proc. Int. Conf. Power Quality: End-Use Applications and Perspectives (PQA'9I), 1991, pp. 171-178. [4]

[10] W. H. Kersting, "Radial distribution test feeders", PES summer meeting, 2000. Available: http://ewh.ieee.org/soc/pes/sacom/ testfeeders.html .

[11] M.A. Hannan, and A. Mohamed, "Modeling and analysis of a 24-pulse dynamic voltage restorer in a distribution system" Research and Development, 2002. SCOReD 2002, pp. 192-195, student conference on 16-17 July 2002. 